\title{
Application of Computer-Assisted Instruction in Horticulture Teaching
}

\author{
Shaohui Wang, Jihong Cheng, Rui Yang, Jinghong-Hao, Li Mei \\ Plant Science and Technology College, Beijing University of Agriculture, Beijing 102206, China
}

\begin{abstract}
The paper focuses on the application of computer-assisted instruction (CAI) in horticulture teaching. The application effect of CAI in teaching is very good due to its features of delight, syllabus-based teaching, interaction and operability. In addition, the suggestions and opinions for further curriculum reform are proposed.
\end{abstract}

Index Terms - Horticulture teaching, multimedia, computer-assisted instruction

\section{Introduction}

Computer-assisted instruction (CAI), as an important component of modern educational technology, is featured by individualized teaching process, diverse expression forms of teaching content and universality of teaching methods. CAI vigorously promotes the reform in teaching methods, teaching tools and teaching contents, constitutes an important part of current teaching reform. Therefore, CAI is listed by the Ministry of Education as one important indicator of undergraduate teaching assessment in higher education institutions.

To satisfy the needs of modern society and respond to challenges, education must cultivate innovative talents. The curriculum reform should be implemented to culture innovative abilities since basic education. Multimedia technology and internet technology have provided favorable conditions and showed new prospect for curriculum reform in the information age [1]. Therefore, the question is how to employ the multimedia teaching to cultivate students' ability of independent learning and initiative exploration, and to create new curriculum, thus to achieve maximum learning effect. Relevant research is of great significance to the implementation of curriculum reform and establishment of new educational patterns.

Horticulture is an important compulsory specialized course for students majored in horticulture science. It is a very practical integrated discipline based on multiple subjects. To adapt to the needs of teaching practice, the teaching mode that combines theoretical teaching with CAI is adopted to realize the visualization of abstract knowledge, so as to strengthen students' sensory awareness, comprehension and to finally improve their professional qualities [2].

\section{Advantages of CAI}

A. Delight

The multimedia courseware that contains pictures, text, voice and videos is used in classroom to stimulate students' interest in the theories. By making a full use of the advantages of multimedia teaching, a large quantity of teaching related knowledge are supplemented to broaden students' thoughts and knowledge and stimulate their interest in learning [3]. For instance, with regard to the knowledge of the growth of Chinese chives, there is only one black-white picture on the textbook. Therefore, it is very difficult for students to grasp the principle of rootstock growth if they cannot even distinguish Chinese chives from wheat. Therefore, the key growth stages of Chinese chives are presented by animated graphics in courseware, thus allowing them to acquire perceptual knowledge. In this way, the students can learn the relevant knowledge very easily.

\section{B. Syllabus-based teaching}

Besides the basic contents specified in teaching syllabus, teaching some new research developments should be added to the teaching courseware to broaden the students' thoughts. However, the class hours are fixed. The limited teaching time should be better used to impart maximum amount of knowledge to students without adding additional class hours. Therefore, teachers are required to prepare their teaching contents carefully before class, while the curriculum team is required to carry out meticulous research on teaching courseware to achieve the visualization of the teaching contents. By this means, the teaching time can be shortened, while the teaching quality is improved. There are a great variety of horticultural plants. The land area is reducing currently. The majority of students studying at Beijing University of Agriculture come from the urban districts and they have no chance to get to know the horticultural plants. Therefore, there is a big barrier in teaching, since the students do not know even the basic horticultural plants. Thus, when it comes to the origin of horticultural plants, the teachers will show students the pictures of some representative horticultural plants, so students can memorize these plants through perceptual knowledge.

\section{Interaction}

Multimedia, which integrates a variety of information transmission means, including texts, graphics, image, audio, animated graphics and video, enables diverse expression forms and creates a sense of reality. Thus, it can stimulate students' interests and create an active classroom atmosphere. As an intuitive and novel teaching means, multimedia teaching is conducive to arousing the students' learning interest [4]. To fully tap the students' potentials, the unconscious learning activities should be combined with conscious learning activities. This method not only enriches the teaching contents, but also enlivens the classroom atmosphere. Moreover, the students' enthusiasm for learning can be mobilized and the creative thinking can be inspired. To 
strengthen the learning initiative, the interaction should be highlighted between teacher and students. Here some easy contents can be learnt by students themselves by requiring them to prepare the teaching courseware. Then teachers and students can exchange their roles in classroom, and an active classroom atmosphere can be created through questions and answers.

\section{Operability}

In conventional instruction methods, the students can see only the teacher, blackboard and several simple teaching tools in classroom. By using only such simple teaching tools, the teacher plays the dominant role in class. During CAI, some techniques should be designed to meet the needs of classroom teaching, including intuitive buttons, help clues, search function and video hyperlink. These techniques can strengthen students' interests and enrich their knowledge. Thus, the teachers are able to finish the teaching tasks through convenient operation.

Due to the combined expression forms including texts, pictures, sound and images, the CAI, which draws a large quantity of resources from the Internet, can greatly enhance students' interest, perception and comprehension. This technique represents a major breakthrough in classroom teaching. However, it is not an easy job to properly apply the multimedia-assisted instruction.

\section{Disadvantages of CAI}

\section{A. Reducing}

The teaching effect. If the teacher overemphasizes the advantages of multimedia-assisted instruction and only seek audio-visual effects, the CAI may even play a negative role in teaching. Thus, the teaching skills implemented by the teacher in classroom instruction may be restricted because the students' dominant position, teachers' guiding role and emotional exchange are all ignored [5].

\section{B. Focusing}

On the huge capacity of knowledge. In order to impart a large amount of knowledge, some teachers just show students a pile of teaching materials without any key points. The students cannot grasp the basic teaching contents. In this case, the teachers fail to attract students' attention, but and the efficiency of classroom teaching will be reduced. Moreover, it is not favorable to establish students' knowledge framework. The classroom interaction may gradually lose its positive effect over time. The students' learning enthusiasm may be drenched.

\section{Overconfidence of the multimedia}

Multimedia instructions bring convenience to teachers in lesson preparation. Some teachers use the same teaching courseware for several years, without adding new contents before class. In addition, some teachers have the habits of reading the contents displayed on computer without explanation. The teachers are not serious enough about teaching.

In conclusion, multimedia-assisted teaching is an assistant instruction means. The use of multimedia in teaching is one of the basic skills for teachers. Teachers should make a full use of multimedia in teaching to improve their teaching. Teachers should reasonably employ the teaching means of multimedia to enhance the students' ability to analyze and solve problems and to cultivate their logical thinking and innovation ability.

\section{References}

[1] Mu Zhibing. Animation Design Idea in Courseware Preparation. Journal of Longdong University (Natural Science Edition), 2006, 16(4):101-102

[2] Xu Caiqin. Several Ideas on Context Setting in Information Technology Teaching. Net Time, 2008, (14):105

[3] Li hanlan. Giving Full Play to the Supportive Role of Computer Multimedia Technology in Chemistry Teaching. Examinations Weekly, 2008, (29):162-163

[4] Zhang Baoyuan. Analysis on Several Problems in Multimedia Teaching. Technology Information, 2008,(12):558

[5] Li Ji, An Investigation and Thinking on the Status of Computer-Assisted instruction in Colleges. Education and Vocation. 2005, (17):48-50. 